\title{
A PRIMING METHOD FOR INVESTIGATING THE. SELECTION OF MOTOR RESPONSES *
}

\author{
David A. ROSENBAUM
}

Bell Laboratories, USA

Sylvan KORNBLUM

The University of Michigan, USA

Accepted November 1981

\begin{abstract}
We describe a priming method for investigating the mechanisms underlying the selection of motor responses. The empirical question addressed with the method is how the choice reaction time for a response depends on its relationship to a response that the subject was primed to perform. We explore the method in a study of manual response selection where we investigate the effects of requiring that two possible responses use the same finger or hand. A requirement of the method that subjects get ready to perform primed responses only - is not met in some conditions of the experiment. When the two possible responses are made with different hands, it appears that multiple response preparation occurs prior to detection of the reaction signal, whereas when the two possible responses are made with different fingers of the same hand it appears that advance preparation is limited to a single response. This finding implies that subjects engage in different kinds of response preparation depending on the relationship between the alternative possible responses. We discuss the implications of this hypothesis for the priming method introduced here as well as for theories of response selection generally.
\end{abstract}

\section{Introduction}

The successful execution of a voluntary body movement presents an intriguing problem to the student of behavior: out of all the movements the actor is capable of performing, how are the movements that he or she performs on a given occasion selected? For example, if a person moves his right arm away from the frontal plane of the body and over a

* Preparation of the paper benefitted from suggestions made by Judith F. Kroll and Saul Sternberg.

Mailing address: D.A. Rosenbaum, School of Language and Communication, Hampshire College, Amherst, MA 01002, USA.

0001-6918/82/0000-0000/\$02.75 1982 North-Holland 
distance of $20 \mathrm{~cm}$, how is it that the right arm is used rather than the left, that the movement proceeds away from the frontal plane rather than towards it, and over $20 \mathrm{~cm}$ rather than, say, $50 \mathrm{~cm}$ ? Somehow, decisions must be made that allow the executed movement to have the properties that it does.

In this paper we describe an experimental method to investigate how such decisions are made. First, we present in greater detail some of the questions that the method is designed to answer. Then we describe the method itself, which we call the response priming method, illustrating its use with an experiment on the selection of finger responses. The results of the experiment are shown to agree with the results of several earlier experiments on manual responses but to conflict with the results of other experiments. We show that the differences in results can be traced to differences in the nature of the subject's response uncertainty in the two classes of experiments. We propose that under different conditions of response uncertainty, subjects may adopt different methods of response preparation, and we tentatively identify two such methods here. We end the paper by considering the implications of our proposed distinction for future studies of movement selection, including studies that use the response priming method.

\section{Questions about movement selection}

Movements, like stimuli, can be assumed to differ with respect to values or features on independent dimensions. For example, in the case of the arm movement mentioned at the beginning of this paper, one can describe the movement in terms of its defining values on the three dimensions of arm, direction, and extent. However, just because these dimensions may be useful for describing the movement, it does not follow that they are actually used in selecting the movement. Dimensions that are actually used in movement selection can be referred to as control dimensions, and it can be asserted that one of the central aims of the study of movement selection is to find out what the control dimensions are (see Brooks 1978; Hayes and Marteniuk 1976).

A second major aim of the study of movement selection is to learn the details of the time course of the movement selection process. Are the values defining a movement specified serially or in parallel? If the specification is serial, are the values specified in a fixed or variable order? Are the times to specify different values on a given control 
dimension the same or different, and are the times to specify values on different control dimensions generally the same or different? Is the time to specify a value on one control dimension independent of which other values have been or are being specified on other control dimensions? And do answers to any of the above questions depend on the behavioral context in which the selected movement is performed (e.g., the identities of other movements that have just been performed), the skill level of the actor, or other factors? If the above questions could be answered, even for a few movements, one could claim to have gained access to the history of those movements.

\section{The movement precuing technique}

How can one address these questions experimentally? One convenient approach is to make use of the choice reaction time (RT) procedure, where each of the possible responses has a unique reaction signal associated with it. When one of the reaction signals is presented the subject is required, under normal conditions, to perform the associated response as quickly as possible. The choice RT is assumed to include, among other things, the time to select the required response (Sanders 1980; Smith 1968; Sternberg 1969) [1].

Recently, one of us (Rosenbaum 1980) introduced a variant of the choice RT procedure that was designed to reveal the detailed characteristics of the time course of the response selection process. In experiments using this movement precuing technique the possible responses are chosen by the experimenter to differ orthogonally with respect to $v$ values on each of $d$ dimensions, resulting in a set of $v^{d}$ responses. As in traditional choice RT experiments, a unique reaction signal is associated with each response and when a reaction signal is presented, the subject is required to perform the corresponding response as quickly as possible. Shortly before the reaction signal appears, however, advance information is given about some, none, or all of the defining values of

[1] We use the term "response" to refer to an equivalence class of movements that serve the same purpose in an experiment (e.g., depression of a particular key). With suitable experimental control, the various movements comprising the equivalence class of a response can be made to be minimally different, although it would be inappropriate to assume that the movements are identical, since fluctuations in the spatial and temporal aspects of repeated, simple movements, such as hand cranking (Glencross 1973) and finger tapping (e.g., Wing 1980), arc invariably observed. 
the response that will be designated by the reaction signal. The RT to make the response when the reaction signal appears is assumed to include the time to specify those movement values that could not be specified in advance, either because they were not disclosed in advance (i.e., were not precued) or because the advance information that was provided could not be used (e.g., because the advance information concerned a dimension for which values could not be specified until information about another dimension, that was not precued, was also given). It will be noted that the movement precuing technique is similar to the partial advance information procedure originally developed by Leonard (1958) and used subsequently by Shaffer (1966) and Kantowitz and Sanders (1972). The movement precuing technique differs from the partial advance information procedure in that it has more data-analytic elaborations and a greater emphasis on motor preparation. The movement precuing technique has so far been used to investigate the selection of manual responses differing with respect to arm, direction, and extent (Dixon and Just 1980; Goodman and Kelso 1980; Rosenbaum 1980), dircction and extent only (Larish 1980), direction, extent, and duration (McCracken 1979), and finger and hand (Miller 1982). The method has also been applied to the study of reflex modulation prior to leg movements (Requin 1979) and decisions concerning the side of the body (right or left), limb (arm or leg), and direction (forward or backward) of simple, ballastic movements (Rosenbaum 1978). A general review of the applications of the movement precuing technique is given in Rosenbaum (in press).

Two caveats must be observed in interpreting data from movement precuing experiments, however. The first is related to the fact that distinct reaction signals are used for the possible responses. When a precue facilitates performance, it is possible that the locus of the facilitation is the stimulus identification stage rather than the response selection stage. For example, subjects may use precues to form expectancies about which reaction signals may appear. If RTs are more reduced by one type of precue than another, it may be because stimulus expectancies can be developed more easily on the basis of one type of precue than another. Evaluating this hypothesis requires control experiments or manipulations of the mappings between reaction signals and responses (see Goodman and Kelso 1980; Rosenbaum 1980).

A second caveat concerns the nature of the subject's response preparation following the presentation of the precue. It is possible that 
subjects ready several of the experimentally defined responses allowed by a precue (e.g., all responses made with the right hand if a precue indicates that the right hand will be used) and then select from this set the response that is designated by the reaction signal. Differences in RTs associated with different types of precues could then be attributed to differences in the discriminability of readied responses, where the discriminability depends on the ensemble of responses that is readied (see Rosenbaum 1980, for a more detailed discussion and relevant control experiment).

\section{The response priming technique}

The response priming technique is designed to overcome the above limitations. The technique is inspired by the priming methods developed by LaBerge et al. (1970) and Posner and Snyder (1975). In essence, the approach consists of creating a bias for one response, the primed response, and then occasionally requiring the subject to perform some other response. The main independent variable is the relationship between the two possible responses. The main empirical question is how the RT of the required response depends on its relationship to the primed response. This question has been addressed by Rosenbaum (1977), Larish (1981), and Sternberg (1981).

Before we show how the response priming method can be used to investigate the time course of response selection, we should explain why we think the method can overcome the difficulties associated with the movement precuing technique. First, with regard to the possibility that subjects may use a variety of response preparation strategies, the response priming method is specifically designed to induce subjects to adopt fixed preparation strategies, particularly when a very strong bias is created for one response. If a strong response bias exists (i.e., if the subject has a strong expectation that the primed response will be required) it is in the subject's interest to ready the primed response. Moreover, if readying any other response would increase the latency of the primed response, it is also in the subject's interest to ready only the primed response. (Later, we shall describe methods for determining whether primed responses alone are readied.)

With regard to the possibility that stimulus identification may account for movement precuing effects, in a response priming experiment it is possible to greatly simplify the subject's stimulus-processing require- 
ments and thereby reduce the likelihood that stimulus-processing actually accounts for alleged response effects. For example, suppose that in a response priming experiment only two responses are tested in a block of trials and the same two reaction signals (e.g., a high and low tone) are used in all blocks. The subject's perceptual task would presumably be the same in all blocks, namely, to decide which of the two reaction signals was presented. Thus, if the RT for a response turned out to depend on its relationship to the other response that could be tested in the block, one would be hard-pressed to explain this effect in non-motor terms. (One possible explanation that is not purely motor, however, would be that the compatibility of the reaction signals (which are fixed) to the responses (which vary across conditions) was not constant. A straightforward way of testing this hypothesis would be to use different reaction signals. If the effects of type of response pair depended on stimulus-response compatibility, different response-pair effects would be obtained if the compatibility relations were changed by the introduction of other stimuli.)

In the section that follows we describe, in more detail, how the response priming method can be used to draw inferences about the time course of response selection. For convenience, we assume that if the probability of requiring a primed response is very high and there are high incentives for responding quickly and accurately when a primed response is required, the subject will consistently ready only the primed response.

Consider an experiment in which there are four possible responses which differ orthogonally with respect to two values on two dimensions. Suppose the responses are key presses made with the ring or index finger of the left or right hand. These responses differ with respect to two values on the "finger dimension" (viz., ring or index) and two values on the "hand dimension" (viz., left or right). Suppose that every possible pair of these responses is tested in a block of trials and that each response is tested equally often when the response with which it is paired is primed. The resulting design is shown in table 1 . The primed and required response may either be the same $(s)$ or they may differ with respect to finger $(f)$, hand $(h)$, or both finger and hand $(f h)$.

Suppose now that each cell in the table is filled with an observed mean RT. The first aspect of the data that one would want to check is that the observed mean RTs in the $s$ cells are shorter than in the other cells; this inequality would be expected if subjects had readied the 
Table 1

Design of response priming experiment. ${ }^{\text {a }}$

\begin{tabular}{lllll}
\hline Required response & \multicolumn{4}{l}{ Primed response } \\
\cline { 2 - 5 } & $\mathrm{Lr}$ & $\mathbf{L i}$ & $\mathbf{R i}$ & $\mathrm{Rr}$ \\
\hline $\mathbf{L r}$ & $s_{1}$ & $f_{1}$ & $f h_{1}$ & $h_{1}$ \\
$\mathbf{L i}$ & $f_{2}$ & $s_{2}$ & $h_{2}$ & $f h_{2}$ \\
$\mathbf{R i}$ & $f h_{3}$ & $h_{3}$ & $s_{3}$ & $f_{3}$ \\
$\mathbf{R r}$ & $h_{4}$ & $f h_{4}$ & $f_{4}$ & $s_{4}$
\end{tabular}

a Primed and required responses are made with the left ring (Lr) finger, left index (Li) finger, right index $(\mathrm{Ri})$ finger, or right ring $(\mathbf{R r})$ finger; the two types of responses are either the same $(s)$ or differ with respect to finger $(f)$, hand $(h)$, or finger and hand $(f h)$. Common subscripts refer to common required responses.

primed responses. In anticipation of this requirement of the data, one might take pains to insure that the mean RTs in condition $s$ in fact arc shorter than in the other conditions; e.g., payoffs or other schemes might be used to encourage fast RTs in the $s$ trials. A second aspect of the data that one would want to check also pertains to the $s$ cells, although it is not explicitly represented in table 1 . A consequence of the design presented in table 1 is that each response, when primed and then required, is paired with each of the other three responses in different conditions of the experiment. As a result, one would actually obtain three separate estimates of the mean RT for a response when it was required after being primed. For example, the left ring response $(\mathrm{Lr})$ would be primed and then required when the other possible response was the left index response ( $\mathrm{Li}$ ), the right index response ( $\mathrm{Ri}$ ), or the right ring response $(\mathbf{R r})$. If a primed response were readied to the same degree regardless of which other response could be tested, the latency of that primed response would be expected to be independent of the identity of the other possible response. If a dependency were found, therefore, it would weaken the assumption of consistent readying of primed responses (although such a result would not in itself be uninteresting).

Suppose now that one was satisfied that subjects had in fact readied primed responses to an equal degree in all response contexts, and also that the assumption was thereby accepted that nonprimed responses 
were not readied. (Note that we are speaking here of the readying of responses prior to detection of the reaction signal.) Mean RTs in the non- $s$ cells of the matrix could then be used to make inferences about whether the subject's state of readiness for the primed response systematically affected the production of the nonprimed response. If the latency of the nonprimed response depended on its relationship to the primed response, one could conclude that the subject's readiness for one response systematically influenced the production of other responses and, moreover, that response production, in general, was not independent of context.

It would next become possible to address questions about the detailed characteristics of the response (re)selection process. Recall that one of the questions raised earlier about movement selection was whether the time required to specify different values on one dimension are the same or different. This question could be addressed in the present context by considering mean RTs for required responses that differ from primed responses with respect to different values on one dimcnsion. For example, the cells labelled $f_{1}$ and $f_{4}$ in table 1 correspond to conditions in which the ring finger has to be used after an index-finger response is primed; and the cells labelled $f_{2}$ and $f_{3}$ correspond to conditions in which the index finger has to be used after a ring-finger response is primed. To determine whether the time needed to specify the ring finger differs from the time needed to specify the index finger, one could compare the following two quantities:

$F_{i r}=\frac{1}{2}\left[\left(f_{1}-s_{1}\right)+\left(f_{4}-s_{4}\right)\right]$

$F_{r i}=\frac{1}{2}\left[\left(f_{2}-s_{2}\right)+\left(f_{3}-s_{3}\right)\right]$

where $F_{i}$, denotes the time to change from the index finger to the ring finger, and $F_{r i}$ denotes the time to change from the ring finger to the index finger. By subtracting the $s$ terms, we remove possible differences in the execution latencies of the four required responses [2]. The sign of the difference between $F_{i r}$ and $F_{r i}$ indicates which type of transition

[2] In using the subtractions for this purpose, we are making a "pure insertion" assumption (Sternberg 1969) about latency differences among responses. We are assuming, principally for convenience, that part of the latency difference between two motor responses is accounted for by differences in execution latencies alone, which are present regardless of the condition $(s, f, h$, or $f h$ ) in which the responses are produced. 
takes longer, and the size of the differences indicates by how much. (Of course, similar comparisons could be made for hand transitions and finger-plus-hand transition.)

It will be recalled that another question about movement selection introduced earlier was whether values on different dimensions are specified independently, that is, whether the time to specify a value on one dimension depends on values on other dimensions that have been or are being specified. The quantities presented in equations 1 and 2 can be used to address this question. Suppose, for example, that the quantities $\left(f_{1}-s_{1}\right)$ and $\left(f_{4}-s_{4}\right)$ were found to differ significantly. As can be seen in table $1,\left(f_{1}-s_{1}\right)$ provides an estimate of the time to change from the ring to the index finger of the left hand, whereas $\left(f_{4}-s_{4}\right)$ provides an estimate of the time to change from the ring to the index finger of the right hand. If these two quantities differ, the implication is that the time to change from the ring to the index finger depends on the hand to be used (at least when hand does not also have to be changed). To find out whether the dependency also obtains when a change of hand is necessary, one could ask whether $\left(f h_{1}-s_{1}\right)$ differs significantly from $\left(f h_{4}-s_{4}\right)$. One's belief about whether a difference between these quantities is attributable to differences in finger-changing times or hand-changing times could be tempered by also considering $\left(h_{1}-s_{1}\right)$ and $\left(h_{4}-s_{4}\right)$, which are estimates of the times to change to the left and right hands, respectively, when a ring-finger response is required. The analyses outlined above could of course also be generalized to other dimensions.

Still another question about the value-specification process that was raised earlier was whether the values defining a forthcoming movement are specified serially or in parallel. For present purposes, we assume that two values are specified serially if the time to specify both values equals or exceeds the sum of the times to specify each value individually. For the hypothetical experiment outlined in table 1, the question of seriality applies to the specification of finger plus hand $(f h)$. We have already shown how one can obtain estimates of the time to effect a particular type of finger change (equations 1 and 2), and it is easy to see how one can obtain estimates of hand-change times and finger-plushand-change times for a given response. For the required left ring response, for example, the quantity $H_{1}=h_{1}-s_{1}$ provides an estimate of the time to change from the right to the left hand, and the quantity $F H_{1}=f h_{1}-s_{1}$ provides an estimate of the time to change from the left 
to the right hand and from the index to the ring finger. If seriality holds for the specification of the left ring finger, one would expect the statement $F H_{1} \geqslant F_{1}+H_{1}$ to be true. Comparable analyses for all of the responses would be needed to evaluate the generality of the seriality conclusion.

\section{An illustrative experiment}

Our discussion of the response priming method has been highly abstract up to now. We have only considered a hypothetical experiment and we have not discussed how the method can be implemented experimentally. We now turn to the latter issue.

Experimentally, biases can be created through the use of instructions, payoffs, manipulations of response probabilities, and presentations of priming signals. There are, moreover, two major types of choice procedures that can be used: (1) the serial choice RT procedure, where the reaction signal for the response on trial $n$ is presented immediately after the response performed on trial $n-1$ (see Kornblum 1973); and (2) the discrete-trials choice RT procedure, where there is usually a relatively long delay between successive trials, often with feedback provided to the $S$ about performance in the last trial. The discrete-trials choice RT procedure has the advantage that it more readily allows for the presentation of priming signals. Principally for this reason we used a discrete-trials choice RT procedure (with priming signals) here. The experiment used the four responses discussed in the introduction of the response priming method, namely, button presses made with the ring and index fingers of the left and right hands.

\section{Method}

The four possible responses, left and right index and middle fingers, generate six possible pairs of responses. Each pair was tested in a separate block of trials. The buttons were connected to microswitches. Approximately $120 \mathrm{gm}$ of static force was required on each button in order to close its associated microswitch, and each button had to be depressed approximately $2 \mathrm{~mm}$ for its associated microswitch to be closed. The reaction signals were a high-pitched $(1000 \mathrm{~Hz})$ tone and a lower-pitched $(600 \mathrm{~Hz})$ tone, each of which was presented for $50 \mathrm{msec}$ over earphones. Auditory stimuli were used because they are known to yield faster RTs than visual stimuli; the pitch dimension was used on the assumption that it was neutral with respect to the left-right dimension. (This assumption was later corroborated by the results.) During a block of trials all four relevant fingers rested on the buttons at all times. There were two groups of $S \mathrm{~s}$, with three right-handed women between age 30 and 40 , randomly assigned to each group. For one group the high tone was consistently assigned to the left response in the pair to be tested and the low tone was consistently assigned to the right response; for the other group the assignments were reversed. This stimulus-response (S-R) assignment scheme allowed each reaction signal to be assigned equally often to each response in each response-pair condition. 
Fig. 1 illustrates the major aspects of the procedure. At the start of the trial, one of the two reaction signals was presented as a prime. One second later, either the same signal was presented again (with probability 0.75 ) or the other signal was presented (with probability 0.25 ). $S$ s were told to become as highly prepared as possible to make the response designated by the prime, not only because the prime would tend to be repeated, but also because bonuses would depend more heavily on speed in trials where primed and required responses matched than in trials where primed and required responses did not match.

Each pair of responses was tested in four consecutive blocks of 22 trials each, with the first two trials of each block serving as warm-ups only. The two responses in each response pair were primed equally often within the four blocks. All six sets of four blocks, corresponding to all six response pairs, werc run in each session. Each $S$ attended one $1 \mathrm{hr}$ session on each of three consecutive days.

\section{Results}

\section{Reaction times}

Table 2 shows mean RTs for correct responses obtained on the last day of the experiment, averaged over the two groups of $S$ s. The four main columns differ with respect to the relationship between the primed and the required response. About 578 observations are represented in each cell of the $s$ (same) column and about 65 observations are presented in each of the other three columns. An analysis of variance showed that the effect of required response (i.e., required finger) on mean RT was not

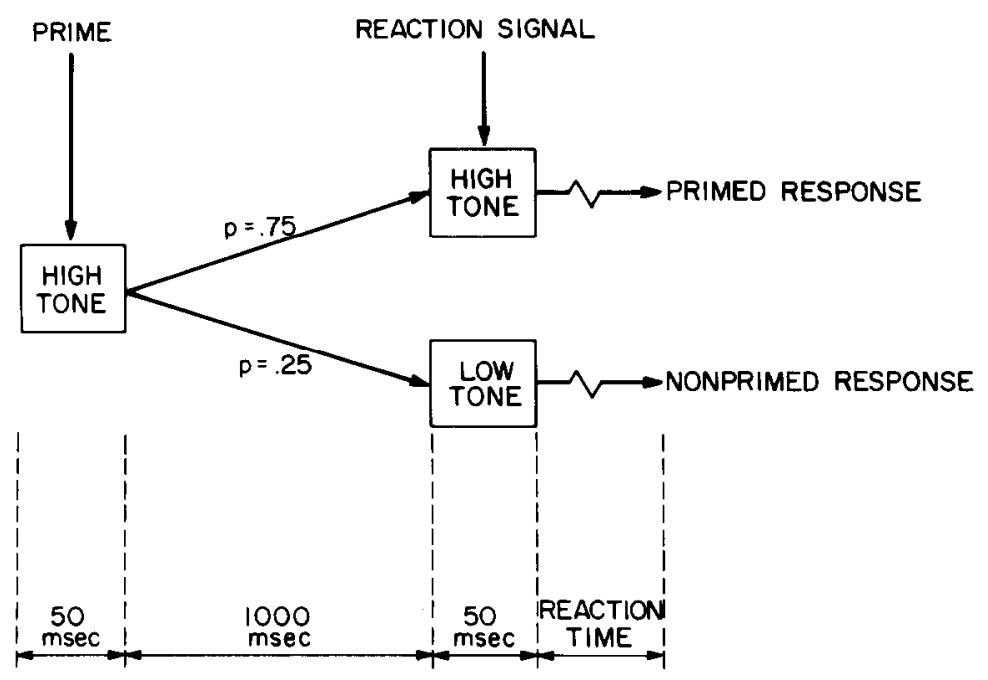

Fig. 1. Procedure used in the response priming experiment. 
Table 2

Mean RTs on the last day of the experiment for each required response depending on how it differed from the primed response.

\begin{tabular}{llllll}
\hline $\begin{array}{l}\text { Required } \\
\text { response }\end{array}$ & \multicolumn{2}{l}{ Dimension of difference } & Mean \\
\cline { 2 - 5 } & $s$ & $f$ & $h$ & $f h$ & \\
\hline $\mathrm{Lr}$ & 240 & 390 & 354 & 353 & 334 \\
$\mathrm{Li}$ & 249 & 390 & 345 & 348 & 333 \\
$\mathrm{Ri}$ & 249 & 367 & 368 & 362 & 337 \\
$\mathrm{Rr}$ & 244 & 384 & 341 & 349 & 330 \\
Mean & 246 & 383 & 352 & 353 & 334 \\
\hline
\end{tabular}

statistically significant, $F(3,10)=0.466 p>0.71$. Nor were the effects of S-R mapping, $F(1,8)=2.69, p>0.15$, or any interactions involving S-R mapping. The effect of the relationship between primed and required response was statistically significant, $F(3,9)$ $=12.16, p<0.001$; even when a separate analysis of variance was carried out which excluded $s$ values, the effect of the relationship between primed and required response was still significant, $F(2,9)=5.12, p<0.05$. For all $S$ s, mean RTs were shortest when the required response was the same as the primed response. Also for all $S$ s, mean RTs were longer in the finger-different condition $(f)$ than in the hand-different $(h)$ or finger- and hand-different ( $f h$ ) conditions. However, mean RTs in conditions $h$ and $f h$ did not differ significantly; for three of the six $S$ s, not all in the same S-R mapping condition, mean RTs were longer in condition $f h$ than in condition $h$, for two of the $S \mathrm{~s}$ the reverse was true, and for one $S$ the two conditions had equal mean RTs.

Table 3 shows mean RTs for each response when it was required after being primed, i.e., when the prime was valid, and when the response was paired with each of the other possible responses. In general, the mean RTs for primed responses did not vary with the relationship to the other possible responses with which they were paired, as is seen clearly in the bottom row of the table.

\section{Errors}

The mean error rate was $2.1 \%$, and ranged from 0.2 to $4.1 \%$ for individual $S \mathrm{~s}$. On trials in which nonprimed responses were required, $S$ s were more likely to respond correctly if the required response used a different hand from the hand that was primed than if it used the same hand. Moreover, $S$ s were equally accurate regardless of whether the nonprimed, required response used the finger homologous or nonhomologous to the primed finger of the opposite hand.

\section{Incidental observations}

On several occasions, instead of just performing the required primed response, $S \mathrm{~s}$ also produced the other possible response shortly afterwards. Unfortunately, we had not foreseen this phenomenon, and so the computer program we used to run this 
Table 3

Mean RTs for each "same" response depending on its relationship to the other possible response. The column labels, $f, h$, and $f h$ refer to the feature(s) distinguishing the validly primed response and its mate.

\begin{tabular}{llll}
\hline Response & \multicolumn{2}{l}{ Relationship } \\
\cline { 2 - 4 } & $f$ & $h$ & $f h$ \\
\hline $\mathrm{Lr}$ & 237 & 248 & 237 \\
$\mathrm{Li}$ & 258 & 242 & 246 \\
$\mathrm{Ri}$ & 243 & 250 & 255 \\
$\mathrm{Rr}$ & 245 & 240 & 249 \\
Mean & 246 & 245 & 247 \\
\hline
\end{tabular}

experiment did not allow for the detection of additional responses after correct responses were performed. Nevertheless, by watching a light panel connected to the response buttons, the experimenter could see when extra responses were made. Using this rather crude observation procedure, it was noted that extra responses occurred on about $6 \%$ of the trials in which primed responses were required and correctly performed. Of the extra responses, only one was a response that used the same hand as the primed response, and of the remaining extra responses, the finger homologous to the primed-response finger was used about as often as the nonhomologous finger.

\section{Discussion}

Two aspects of the mean RT data are consistent with the requirements of the response priming method for making detailed inferences about the response selection process. First, mean RTs were shorter for primed than for nonprimed responses. This result is consistent with the requirement that subjects get ready to perform primed responses. Second, mean RTs for primed responses did not depend on the identity of the other possible response. This result is consistent with the requirement that primed responses are readied in a consistent fashion, i.e., to an equal degree, in all experimental contexts.

Some aspects of the results, however, preclude the use of all the inferential procedures afforded by the response priming method. In particular, mean RTs were longer when finger alone had to be changed $(f)$ than when both finger and hand had to be changed $(f h)$. At first, this may appear to be an odd result: why would the time to change a 
single value (or what is assumed to be a single value) be longer than the time to change this value and one more in addition? Other aspects of the data give a clue, as do the subjects' introspective reports. Subjects reported that when the two possible responses in a block of trials were made with different hands, both responses could be held in readiness until the reaction signal was presented, but when the two possible responses used the same hand only the primed response could be readied. These reports are supported by the incidental observations and the error data that were reported above.

Later in this paper we consider the implications of this outcome for theories of response selection. Now, however, we turn to the inferential procedures afforded by the response priming method, and make use of the procedures which are warranted by the data.

In table 4 we present adjusted mean RTs for responses that were required after not being primed; note that each adjusted mean RT is the difference between the obtained mean RT and the mean RT for the same response when it was required after being primed. Recall that earlier we proposed carrying out such subtractions to remove possible differences among execution latencies for the four responses. Recall also that insofar as our technique is similar to Posner and Snyder's (1975) method, as we indicated before, each adjusted mean RT may be viewed as the sum of the facilitating and inhibiting effects of the prime in the $s$ and the other conditions, respectively.

Consider first the entries in column $f$, where we present the adjusted mean RT for each response when it differed from the primed response

Table 4

Adjusted mean RTs for each nonprimed, required response depending on its relationship to the corresponding primed response. ${ }^{\text {a }}$

\begin{tabular}{llrr}
\hline $\begin{array}{l}\text { Required } \\
\text { response }\end{array}$ & \multicolumn{2}{l}{ Relationship } & \multicolumn{1}{l}{} \\
\cline { 2 - 4 } & $f$ & $h$ & 113 \\
\hline $\mathrm{Lr}$ & 150 & 114 & 99 \\
$\mathrm{Li}$ & 141 & 96 & 113 \\
$\mathrm{Ri}$ & 118 & 117 & 105 \\
$\mathrm{Rr}$ & 140 & 97 & \\
\hline
\end{tabular}

a Each entry is the difference between the mean RT in the indicated nonprimed condition and the mean RT for the same response when it was required after being primed. 
with respect to finger only. With no evidence that these responses were readied when their primed response-mates were readied, we assume that these nonprimed responses in fact were not readied in the period between the priming and reaction signals. The values in column $f$ then allow us to conclude that on the average finger changes took longer for the left hand than the right.

Consideration of the values in columns $h$ and $f h$ also allows for some tentative conclusions about the selection of nonprimed though readied responses. First, consider the fact that the values in columns $h$ and $f h$ are approximately equal for all responses; the differences between the values in the two columns do not approach statistical significance for any response. If it is assumed that the values in these two columns are indicative of the degree to which the responses could be readied, the equivalence implies that a finger response can be readied to an approximately equal degree regardless of whether it uses the same finger or a different finger from a primed response that uses the other hand. This conclusion suggests independence between the hands, at least in this sort of task.

A second conclusion is permitted by the data in columns $h$ and $f h$, although it does not admit of such a ready interpretation. The conclusion is that for the left hand, the index finger could be readied more easily than the ring finger, whereas for the right hand, the ring finger could be readied more easily than the index finger. Why this might be is unclear at this time. Conceivably, the effect may reflect a directional or spatial bias toward the right which could have resulted from some peculiar aspect of the experimental procedure, such as the consistent mapping of the high and low tones (which served as priming and reaction signals) to responses on the right or left.

\section{Relations to earlier results}

How do the data from this experiment relate to findings from previous studies? Our hypothesis that two responses could be readied if they used opposite hands is consistent with the conclusion of a choice RT study by Kornblum (1965). In one condition of Kornblum's study subjects chose between a right middle-finger response and a right index-finger response (same-hand condition); this condition is analogous to the condition of the present experiment in which the two possible responses were made with the index or ring finger of one hand. 
In the other condition of Kornblum's (1965) study, subjects chose between the right middle-finger response and a left index-finger response (different-hand condition); this condition is analogous to the condition of the present experiment in which the two possible responses were made with two fingers of opposite hands. In Kornblum's experiment the mean RT for the common, right middle-finger, response was $310 \mathrm{msec}$ in the same-hand condition, and $282 \mathrm{msec}$ in the different-hand condition. This outcome is similar to the finding in the present experiment that when the two possible responses used the same hand, latencies were longer than when they used opposite hands. Kornblum (1965) inferred from his result that ipsilateral (same-hand) responses experience more competition and/or inhibition than contralateral (different-hand) responses. Thus, Kornblum's interpretation is consistent with the one offered here, namely, that within- and between-hand responses differ in the degree to which they can simultaneously be held in readiness for immediate execution. The results obtained here show, in addition, that the superiority of different-hand responses is present even if a bias is introduced for one of the responses. Furthermore, the present results indicate that the superiority of different-hand responses is about the same regardless of whether the two possible responding fingers are homologous or nonhomologous.

It is interesting to note that in two studies of serial choice RT tasks, where the signal to respond is presented shortly after the last response and there is no intervening warning signal, mean RTs have been found to be faster for within-hand transitions than for between-hand transitions (Kornblum 1973; Rabbitt 1968). Why have within-hand transitions been found to take less time than between-hand transitions in serial choice RT tasks, whereas response latencies have been found to be longer for between-hand choices than within-hand choices in discrete-trials choice RT experiments? One rather mundane possibility is that the serial choice result is simply an artifact of the way that Kornblum's (1973) and Rabbitt's (1968) serial choice experiments were run. Responses in their experiments were signaled by lights placed directly above corresponding fingers, so that lights presented on successive trials were closer together for within-hand transitions than for between-hand transitions. Thus, within-hand transitions may have been faster because subjects could detect and/or identify the reaction signals more easily than when between-hand transitions were required. Unfortunately, we know of no evidence that argues against this interpretation of the Kornblum and Rabbitt results. 
Another, less mundane, possibility is that the within-hand superiority observed by Kornblum (1973) and Rabbitt (1968) is attributable to the serial nature of their task - that is, that responses were made in a continuous rather than a discrete fashion. This hypothesis is weakened, however, by the fact that in studies of skilled typewriting, where subjects either type from printed copy (e.g., Fox and Stansfield 1964) or from memory (Sternberg et al. 1978), times between successive keystrokes are shorter if the keystrokes are made with different hands than if they are made with the same hand. Hence, the serial nature of the serial choice RT task does not predict that within-hand transitions should be faster than between-hand transitions.

Additional evidence which supports the idea that the relative speed of between- and within-hand performance does not depend on whether a serial- or discrete-trials procedure is used comes from a study by Miller (1982). Miller used the movement precuing technique in a study concerned with the specification of finger and hand. Miller's responses were button presses made with the right or left middle or index finger. Before presenting a reaction signal that indicated which of the four responses would be required, Miller gave advance information about the finger, hand, finger and hand, or no defining features of the response that was about to be tested. Over a wide range of procedures for presenting precues and reaction signals, Miller found that when only two responses were permitted by the precue, RTs were shorter when the two responses used fingers of the same hand than when they used fingers of opposite hands. This result is the opposite of the result obtained in the priming experiment reported here and in Kornblum's (1965) discrete-trials choice experiment.

Why then have opposite within- and between-hand effects been observed in different experimental settings? To address this question, it is useful to consider what factors may have differentiated the experiments that yielded within-hand and between-hand superiority. Consider first the precuing experiments of Miller, in which within-hand superiority was observed, versus the choice experiments reported here and by Kornblum (1965), in which between-hand superiority was observed. In Miller's study, subjects were not tested on a fixed pair of responses within a block of experimental trials, and the foreperiod from the precue to the reaction signal was varied. By contrast, in Kornblum's (1965) experiment and in the experiment reported here, the pair of responses that could be tested in a block of trials was fixed and a 
constant foreperiod was employed. Thus, in Kornblum's (1965) experiment and in the experiment reported here there was less response uncertainty - or more properly, response pair uncertainty - than in Miller's (1982) experiment.

A difference in response uncertainty also characterizes the serial choice experiments of Kornblum (1973) and Rabbitt (1968) and the studies of typing reported, for example, by Sternberg et al. (1978). In the serial choice experiments, subjects had no information about which response would be required after a given response was tested. However, in the typing studies, information about the whole set of forthcoming responses was available to the subject. That information about later responses is in fact used by subjects in typing experiments is demonstrated by aspects of their interresponse time (i.e. times between successive keystrokes). Sternberg et al. (1979) observed that times between successive keystrokes made with one hand tended to decline as a change of hand approached. Thus, as with the discrete-trials experiments, it is a fair generalization that in experiments involving serial performance, one observes within-hand superiority (i.e., faster transitions within hands) when there is high response uncertainty, and between-hand superiority (i.e., faster transitions between hands) when response uncertainty is reduced.

\section{Two methods of response preparation}

On the basis of this analysis we would like to introduce the general hypothesis that when people are subjected to different forms or amounts of response uncertainty they are capable of adopting qualitatively different methods of response preparation. We turn now to the question of what those two methods of response preparation might be.

When a person is aware that one of a small number of possible responses will be required when a reaction signal is presented, and also that it is important to minimize the RT for whichever response will be required, it is natural to suppose that the person will make an effort somehow to ready the various possible responses. We shall refer to this method as multiple-response preparation (MRP) and we shall assume that this method was used in the experiment reported here, in Kornblum's (1965) discrete-trials experiment, and in the typing studies of Sternberg et al. (1978).

Under conditions of high response uncertainty, however, it may be 
preferable for the subject not to use multiple response preparation - if, for example, the RT benefit that any one response could enjoy were very small (or possibly even negative). We shall refer to this alternative method as individual-response preparation (IRP) and we shall assume that it was used in the serial choice experiments of Kornblum (1973) and Rabbitt (1968) and in the precuing experiments of Miller (1982).

Too little information is available at this time to permit us to characterize how the MRP and IRP methods might work in detail or why these methods might give rise to opposite within- and between-hand effects. One might speculate that when the MRP method is used, subjects choose between alternative movement representations, and the time required to effect the discrimination increases with the similarity between the representations; "similarity" in this context refers to the number of movement features common to the two representations. When the IRP method is used, however, one might suppose that in performing one response after another the movement representation for the first response is modified as necessary to allow for the second; modifying the movement representation entails changing the features that distinguish the representations of the two responses, where the time to complete the modification increases with the number of distinguishing features (i.e., decreases with the similarity of the representations). This account of the IRP and MRP methods can explain why different within- and between-hand effects have been obtained in the experiments we have referred to here, although the explanation assumes, perhaps somewhat arbitrarily, that responses performed with fingers of the same hand share more features than responses performed with fingers of opposite hands, whether or not they are homologous.

\section{Implications for future studies}

The foregoing discussion can be used to guide choices of experimental procedures for investigating response selection in the future. Recall that in our introduction of the movement priming technique we relied on the assumption that subjects would only ready primed responses. We were frustrated in our effort to exploit the technique fully, however, because we were led to believe that subjects readied nonprimed responses as well as primed responses in some conditions of the present experiment. Now that we have tentatively identified the conditions that give rise to multiple response preparation, however, we can suggest 
experimental procedures that will be more likely to give rise only to preparation of individual responses. Essentially, these are procedures in which there is a high degree of response uncertainty. Of the procedures we have referred to, the one that would probably both result in a high degree of response uncertainty and also allow for the induction of strong response biases is the serial choice RT procedure. The general experimental approach would be to make use of the fact that in serial choice RT experiments the RT for a response tends to decline as the number of successive repetitions of the response increases (i.e., as the response is called for in an increasing number of successive trials); for a review of this effect, see Kornblum (1973). The main independent variable in such a serial choice RT experiment would be the relationship between the response that the subject was primed to perform on a given trial and the response he or she was actually required to perform. We have already seen that in serial choice RT experiments on manual responses the mean $\mathrm{RT}$ for a response made with one hand is affected by whether the preceding response was made with the same or the opposite hand (Kornblum 1973; Rabbitt 1968). Other serial choice RT experiments have uncovered effects of the relationships between responses performed on successive trials (Rabbitt 1965). Thus, we are encouraged that the detailed study of response transition effects in serial choice experiments, using the analytic techniques we have presented here, holds promise as a means of gaining insight into the mechanisms of movement selection.

\section{References}

Brooks, V.B., 1978. 'Motor programs revisited'. In: R.E. Talbott and D.R. Humphrey (eds.), Posture and movement: perspectives for integrating sensory and motor research on the mammalian nervous system. New York: Raven Press.

Dixon, P. and M.A. Just, 1980. Chronometric studies of algorithm selection: the problem of deciding what to do. Unpublished manuscript, Bell Laboratories.

Fox, J.G. and R.G. Stansfield, 1964. Digram keying times for typists. Ergonomics 7, 317-320.

Glencross, D.J., 1973. Temporal organization in a repetitive speed skill. Ergonomics 16, 765-776.

Goodman, D. and J.A.S. Kelso, 1980. Are movements prepared in parts? Not under compatible (naturalized) conditions. Journal of Experimental Psychology: General 109, 475-495.

Hayes, K.C. and R.G. Marteniuk, 1976. 'Dimensions of motor task complexity'. In: G.E. Stelmach (ed.), Motor control: issues and trends. New York: Academic Press.

Kantowitz, B.H. and M.S. Sanders, 1972. Partial advance information and stimulus dimensionality. Journal of Experimental Psychology 92, 412-418.

Kornblum, S., 1965. Response competition and/or inhibition in two-choice reaction time. Psychonomic Science, 2, 55-56. 
Kornblum, S., 1973. 'Sequential effects in choice reaction time: a tutorial review'. In: S. Kornblum (ed.), Attention and performance, IV. New York: Academic Press.

LaBerge, D., P. Van Gelder and J. Yellott, 1970. A cuing technique in choice reaction time. Perception and Psychophysics 8, 51-62.

Larish, D.D., 1980. On the relationship between response organization processes and response programming. Unpublished manuscript, University of Iowa.

Larish, D.D., 1981. How are motor programs restructured? A look at the relationship of arm, direction, and extent. Paper presented at the meeting of the North American Society for Psychology of Sport and Physical Activity, Asilomar, CA.

Leonard, J.A. 1958. Partial advance information in a choice reaction time task. British Journal of Psychology 49, 89-96.

McCracken, H.D., 1979. Programming direction, extent, and duration in aimed hand movements. Unpublished doctoral dissertation, University of Wisconsin-Madison.

Miller, J., 1982. Discrete vs. continuous stage models of human information processing: in search of partial output. Journal of Experimental Psychology: Human Perception and Performance 8, 273-296.

Posner, M.I. and C.R.R. Snyder, 1975. 'Facilitation and inhibition in the processing of signals'. In: P.M.A. Rabbitt and S. Dornic (eds.), Attention and performance, V. London: Academic Press.

Rabbitt, P.M.A., 1965. Response-facilitation on repetition of a limb movement. British Journal of Psychology 56, 303-304.

Rabbitt, P.M.A., 1968. Repetition effects and signal classification strategies in serial choice-response tasks. Quarterly Journal of Experimental Psychology 20, 232-240.

Requin, J., 1979. Personal communication, June.

Rosenbaum, D.A., 1977. Stages of human movement initiation. Unpublished manuscript.

Rosenbaum, D.A., 1978. Selecting the side of the body, limb, and direction of forthcoming movements. Paper presented at the meeting of the Psychonomic Society, San Antonio, TX.

Rosenbaum, D.A., 1980. Human movement initiation: specification of arm, direction, and extent. Journal of Experimental Psychology: General 109, 444-474.

Rosenbaum, D.A., in press. 'The movement precuing technique: assumptions, applications, and extensions'. In: R. Magill (ed.), Memory and control in motor behavior. Amsterdam: NorthHolland.

Sanders, A.F., 1980. 'Stage analysis of reaction processes'. In: G.E. Stelmach and J. Requin (eds.), Tutorials in motor behavior. Amsterdam: North-Holland.

Shaffer, L.H., 1966. Some effects of partial advance information on choice reaction with a fixed or variable S-R mapping. Journ?l of Experimental Psychology 72, 541-545.

Smith, E.E., 1968. Choice reaction time: an analysis of the major theoretical positions. Psychological Bulletin 69, 77-110.

Sternberg, S., 1969. 'The discovery of processing stages: extensions of Donders' method'. In: W.G. Koster (ed.), Attention and performance, II; Acta Psychologica 30, 276-315.

Sternberg, S., 1981. Personal communication, February.

Sternberg, S., R.L. Knoll and C.E. Wright, 1979. The timing of rapid movement sequences in typewriting. Paper presented at the meeting of the Psychonomic Society, Phoenix, AZ.

Sternberg, S., S. Monsell, R.L. Knoll and C.E. Wright, 1978. 'The latency and duration of rapid movement sequences: comparisons of speech and typewriting'. In: G.E. Steimach (ed.), Information processing in motor control and learning. New York: Academic Press.

Wing, A.M., 1980. 'The long and short of timing in response sequences.' In: G.E. Stelmach and J. Requin (eds.), Tutorials in motor behavior. Amsterdam: North-Holland. 